\title{
Case 21-2016: A Man in an Unresponsive State
}

TO THE EDITOR: In the case discussed by Fenves et al. (July 14 issue), ${ }^{1}$ a patient who had a negative test for hepatitis B virus (HBV) surface antigen and a positive test for HBV surface antibodies 6 years earlier (with no previous testing for HBV core antibodies) presents with fulminant hepatic failure due to acute HBV infection, with positive tests for both HBV surface antigen and HBV core IgM antibodies. Reasonable explanations for this presentation are a false positive test for HBV surface antibodies 6 years earlier or infection with a mutant strain of HBV. However, four points, mainly regarding the interpretation of serologic testing for HBV, deserve attention. First, the inactivecarrier state is defined by persistent positivity for HBV surface antigen; thus, a patient with a negative test for HBV surface antigen 6 years earlier cannot be an inactive carrier. ${ }^{2}$ Second, no HBV reactivation of any severity has been described in patients who are negative for HBV surface antigen and do not receive immunosuppression. ${ }^{2}$ Third, HBV DNA is not universally suppressed in patients who have HBV plus a superinfection with HDV; such patients may sometimes have high HBV DNA levels and may even be seropositive for HBV e antigen. In patients who have coinfection with HBV and HDV, HBV replication is present during the acute phase. ${ }^{3}$ Fourth, although the use of antibiotic and antifungal agents has not been consistently associated with increased survival in patients with acute liver failure, these agents are still administered as standard therapy in patients with encephalopathy or coagulopathy and organ failure. ${ }^{4}$

Emmanuel A. Tsochatzis, M.D., Ph.D.

University College London

London, United Kingdom

e.tsochatzis@ucl.ac.uk

George V. Papatheodoridis, M.D., Ph.D.

Laiko General Hospital

Athens, Greece

No potential conflict of interest relevant to this letter was reported.

1. Case Records of the Massachusetts General Hospital (Case 212016). N Engl J Med 2016;375:163-71.

2. European Association for the Study of the Liver. EASL clinical practice guidelines: management of chronic hepatitis B virus infection. J Hepatol 2012;57:167-85.

3. Hughes SA, Wedemeyer H, Harrison PM. Hepatitis delta virus. Lancet 2011;378:73-85.
4. Bernal W, Wendon J. Acute liver failure. N Engl J Med 2013; 369:2525-34

DOI: 10.1056/NEJMc1610229

THE DISCUSSANTS REPLY: We agree with Tsochatzis and Papatheodoridis that inactive carriers have a detectable level of serum HBV surface antigen. The previous positive test for HBV surface antibodies in this patient could have indicated either resolved acute infection or chronic infection that passed through an inactive-carrier phase with eventual clearance of serum HBV surface antigen; either case could result in persistence of the intrahepatocytic replication-competent HBV genome, which could lead to later loss of immune control and reactivation of infection. Such reactivation in the absence of immunosuppression is very rare but has been reported. ${ }^{1}$ Specific laboratory testing would certainly have allowed for a more definitive determination of the presence or absence of superinfection or coinfection with HDV, but the typical inverse relationship of the replicative levels of HDV and HBV in patients with HDV superinfection led to our speculation that acute HDV infection was unlikely in this patient with a high HBV viral load.

Although we agree with a low threshold for initiating antimicrobial agents in critically ill patients with acute liver failure, we do not believe that the use of prophylactic antibiotics should be a standard of care. Guidelines do not recommend universal antimicrobial prophylaxis. ${ }^{2}$ Evidence to suggest a survival benefit is lacking, and certain antimicrobials potentiate liver injury. Future research should aim to define whether a subset of patients could benefit from antimicrobial prophylaxis.

Jay Luther, M.D.

Virginia M. Pierce, M.D.

Massachusetts General Hospital

Boston, MA

Since publication of their article, the authors report no further potential conflict of interest.

1. Kamitsukasa H, Iri M, Tanaka A, et al. Spontaneous reactivation of hepatitis B virus (HBV) infection in patients with resolved or occult HBV infection. J Med Virol 2015;87:589-600. 2. Lee WM, Larson AM, Stravitz RT. AASLD position paper: the management of acute liver failure: update 2011. Alexandria, VA: American Association for the Study of Liver Diseases, 2011. DOI: 10.1056/NEJMc1610229 